\title{
Immunization among Healthcare Workers in India: A Need or a Want
}

\author{
Raja Danasekaran \\ Professor, Department of Community Medicine, Chettinad Hospital \& Research Institute, Chettinad Academy of \\ Research \& Education, Kelambakkam, Tamil Nadu
}

Immunization is a highly effective way for prevention of some major infectious diseases. Healthcare workers (HCWs) are at greater risk of exposure as well as spread of vaccine preventable diseases, as they are in constant contact with patients and infectious materials. Many of these diseases still carry a potential for resurgence and can lead to outbreaks. Hence, vaccination programs among $\mathrm{HCWs}$ form an integral part of infection prevention \& control practices, thereby protecting the healthcare personnel from infection and protecting the patients from getting infected. [1] Even in countries with specific vaccination programs for $\mathrm{HCWs}$, the coverage remains very low and the majority are susceptible for vaccine preventable diseases. So, it becomes imperative for a developing country like India and other countries in low-resource settings to have a national vaccination plan for HCWs. [Table 1]. shows the list of vaccines recommended for HCWs by World Health Organization and Center for Disease Control and Prevention. ${ }^{[2,3]}$

The risk of Hepatitis B viral infection from occupational exposure depends on the degree of contact with blood or other body fluids, specifically fluids containing HbeAg. Hepatitis B vaccine produces $>90 \%$ of antibody response after the recommended three dose series at 0,1 and 6 months. Post vaccination serological testing can be done to determine the need for revaccination and to guide about post-exposure prophylaxis. ${ }^{[5]}$ Recent evidences from various studies have proven the fact that influenza leads to nosocomial outbreaks in many settings and the attack rate can be as high as $54 \%$. Such outbreaks also lead to increase in absenteeism among medical staff and disruption of health care. The idea of making annual influenza vaccination mandatory among $\mathrm{HCW}$ s has been proposed by various professional societies. [6] HCWs are recommended to receive a single dose of TdaP followed by revaccination every 10 years with either Td or TdaP. Measles, Mumps and rubella vaccine can be given as 2 doses for those HCWs with no serological evidence of immunity or previous history of vaccination. Varicella spread from HCWs to patients in health settings or even among HCWs who received a single dose of the vaccine have been reported. Ideally, 2 doses of varicella vaccine is recommended which provides 95\% immunogenicity. HCWs such as microbiologists who are dealing with specimens containing meningococci should get vaccinated with meningococcal vaccine, followed by boosters every 5 years. ${ }^{[7]}$

Even though the levels of knowledge regarding the vaccines is better among HCWs than the public, the immunization rate never attains $100 \%$. The various factors that were identified for HCWs not getting immunized are: a) doubts over the safety \& efficacy, b) fear of adverse effects, c) injection related, and d) difficulties in getting vaccines. Also, those non-immune HCWs with contraindications for vaccines need to be identified and should be managed accordingly. ${ }^{[8,9]}$ Strategies which can be adopted for increasing the coverage includes: (a) free vaccination, (b) education to break the myths about vaccination, (c) vaccination sessions on nights as well as weekends, (d) adequate staff

CORRESPONDING AUTHOR: Dr Raja Danasekaran, Professor, Department of Community Medicine, Chettinad Hospital \& Research Institute, Chettinad Academy of Research \& Education, Kelambakkam, Tamil Nadu

E Mail ID: mailraja84@gmail.com

ARTICLE CYCLE: Received: 28/01/2021; Revised: 15/02/2021; Accepted: 01/03/2021; Published:30/06/2021 CITATION: Danasekaran R. Immunization among Healthcare Workers in India: A Need or a Want. J Comp Health. 2021;9(1):50-00. 
and mobile units, etc. ${ }^{[10]}$ Further multi-centred studies are needed to assess the factors which play a decisive role in vaccination among HCWs in different settings. As we have started vaccinating the HCWs for the control of the current COVID-19 pandemic, it becomes an ideal time to frame a National Policy on Immunization of HCWs considering the varied factors and the local needs of the country.

\section{TABLE 1 RECOMMENDED IMMUNIZATION FOR HEALTHCARE WORKERS}

\begin{tabular}{|c|c|c|}
\hline S.No. & Vaccine & Recommendation \\
\hline 1. & Hepatitis B & HCWs who have chances of exposure to blood and blood products at work \\
\hline 2. & Influenza & Annual immunization with a single dose is recommended \\
\hline 3. & $\begin{array}{l}\text { Tdap (Tetanus, } \\
\text { Diphtheria, Pertussis) }\end{array}$ & $\begin{array}{l}\text { one-time dose of Tdap as soon as possible if HCWs have not received Tdap } \\
\text { previously, pregnant HCWs need to get a dose during each pregnancy }\end{array}$ \\
\hline 4. & $\begin{array}{l}\text { MMR (Measles, } \\
\text { Mumps, Rubella) }\end{array}$ & All HCWs to be immune for Measles \& Rubella \\
\hline 5. & Meningococcal & One booster every 3-5 years may be considered for HCWs \\
\hline 6. & Varicella & 2 doses for susceptible HCWs (unvaccinated and no history) \\
\hline 7. & COVID-19 & 2 doses for all HCWs \\
\hline
\end{tabular}

\section{REFERENCES}

1. Centers for Disease Control and Prevention (CDC). Immunization of Health-Care Workers: Recommendations of the Advisory Committee on Immunization Practices (ACIP) and the Hospital Infection Control Practices Advisory Committee (HICPAC). MMWR 1997; 46(RR-18): 1-42.

2. World Health Organization (WHO). Summary of WHO Position Papers - Immunization of Health Care Workers (Updated in September 2020). Available at: https://www.who.int/immunization/policy/Immunization routine_table4.pdf (Last accessed on 24 January 2021)

3. Centers for Disease Control and Prevention (CDC). Recommended Vaccines for Healthcare Workers. Available at: https://www.cdc.gov/vaccines/adults/recvac/hcw.html (Last accessed on 24 January 2021)

4. Melanie D, Amy JB. Vaccines For Health Care Personnel. Mayo Clin Proc. 2019 Oct ;94(10):2127$2141 \mathrm{n}$.

5. Batra V, Goswami A, Dadhich S, Kothari D, Bhargava N. Hepatitis B immunization in healthcare workers. Ann Gastroenterol. 2015 Apr-Jun;28(2):276-280. PMID: 25830669; PMCID: PMC4367220.
6. Perl TM, Talbot TR. Universal Influenza Vaccination Among Healthcare Personnel: Yes We Should. Open Forum Infect Dis. 2019 Apr 17;6(4):ofz096. doi: 10.1093/ofid/ofz096. PMID: 31012441; PMCID: PMC6468130.

7. Haviari $S$, Bénet $T$, Saadatian-Elahi $M$, André $P$, Loulergue $\mathrm{P}$, Vanhems $\mathrm{P}$. Vaccination of healthcare workers: A review. Hum Vaccinlmmunother. 2015;11(11):2522-37.

doi: 10.1080/21645515.2015.1082014. PMID: 26291642; PMCID: PMC4685699.

8. Petek D, Kamnik-Jug K. Motivators and barriers to vaccination of health professionals against seasonal influenza in primary healthcare. BMC Health Serv Res. 2018 Nov 14;18(1):853. doi: 10.1186/s12913-0183659-8. PMID: 30428886; PMCID: PMC6234642.

9. Tomboloni $\mathrm{C}$, Tersigni $\mathrm{C}$, de Martino $\mathrm{M}$, et al. Knowledge, attitude and disinformation regarding vaccination and immunization practices among healthcare workers of a third-level paediatric hospital. Ital J Pediatr 2019; 45:104.

10. Weber DJ, Orenstein W, Rutala WA. How to improve influenza vaccine coverage of healthcare personnel. Isr J Health Policy Res. 2016; 5:61. 